\title{
Teaching Imitation to Young Children with Autism Spectrum Disorder Using Discrete Trial Training and Contingent Imitation
}

\author{
Alice Bravo $^{1}{ }^{[} \cdot$ llene Schwartz $^{1}$ \\ Accepted: 23 September 2021 / Published online: 6 October 2021 \\ ( ) The Author(s), under exclusive licence to Springer Science+Business Media, LLC, part of Springer Nature \\ 2021
}

\begin{abstract}
Imitation is a pivotal skill for children with and without disabilities, serving both learning and social interaction functions for young children. Children with autism spectrum disorder (ASD) frequently experience delays in acquiring this skillset, demonstrating challenges in the ability and/or propensity to imitate the actions of others. Current intervention programs frequently use discrete trial teaching (DTT) or contingent imitation to support imitation development in young children with ASD. This study combined contingent imitation with DTT to teach imitation to individuals who had previously struggled with acquiring this skill. A multiple probe design across three preschool children with ASD was conducted within participants' early learning classrooms. Response to intervention varied across participants, with participants with more advanced imitation skills at study onset demonstrating greater outcomes. Combining contingent imitation with DTT may facilitate the propensity to imitate for individuals who exhibit some appropriate object engagement and are inconsistently imitating others' actions with objects. However, further research using stronger research design is needed to improve the teaching of imitation to young children with ASD who exhibit challenges with this skillset.
\end{abstract}

Keywords Autism · Imitation · Early Intervention · Contingent Imitation · Discrete Trial Teaching

Autism spectrum disorder (ASD) is a developmental disorder that can typically be identified within the first 2 years of life (NIMH, 2018). Diagnostic characteristics of ASD include challenges with social communication skills and the

Alice Bravo

acbravo@uw.edu

1 University of Washington, Box 357925, Seattle, WA 98195, USA 
presence of restrictive interests and/or repetitive patterns of behavior (NIMH, 2018). Delayed development of imitation skills is a major concern in children with ASD, so much so that a lack of imitation skills in young children can trigger ASD screening and diagnostic evaluations and is often one of the first skills targeted during intervention. Individuals with ASD demonstrate more difficulties with imitation when compared to children with fragile $\mathrm{X}$ syndrome, developmental delay, and typical development (Rogers et al., 2003; Stone et al., 1997; Vivanti et al., 2014). While deficits in imitation are not a core diagnostic characteristic of ASD, it does appear that challenges with imitation are distinctive to an ASD diagnosis. This has important implications, given the role that imitation plays in human development.

Uzgiris (1981) described two functions that imitation serves in early childhood: 1) a learning function via which young children develop new abilities through observation and copying of others' actions and 2) a social function via which young children begin interacting with others, engaging in turn-taking and social exchanges. Given the interaction between imitation, social skills, and communication, Toth and colleagues (2006) theorized that imitation may be a beginning skill for infants and toddlers upon which more complex social communication skills may further develop. Others consider imitation to be a pivotal skill, one that opens developmental avenues for children and is used to learn additional skills (Koegel et al., 1999).

Vivanti (2015) describes two possible imitative challenges children with ASD may experience. The first is the ability to imitate, which emphasizes what a child can do and the accuracy of a child's imitative responses and is found to be associated with the individual's level of attending (Vivanti et al., 2014). The second is the propensity (i.e., social motivation) to imitate, which emphasizes what a child actually does (i.e., the individual's spontaneous use of imitation), and is associated with additional social skills such as joint attention (Vivanti et al., 2014). Taken together, these challenges suggest that both the ability and the propensity to imitate should be considered when providing intervention to children with ASD.

\section{Intervention}

Two frequently used evidence-based teaching approaches for imitation are discrete trial teaching (DTT) and contingent imitation. DTT is a behavioral approach consisting of a discriminative stimulus, prompts and prompt fading, reinforcement, and error correction, and has been used to teach imitation to children with ASD since the 1960s (Baer et al., 1967; Lovaas et al., 1967; Metz, 1965). DTT has typically been used to teach imitation in a decontextualized manner, resulting in the criticism that this approach teaches imitation separately from its social communicative context (Ingersoll \& Schreibman, 2006). Because DTT teaches individuals with ASD to imitate actions in response to an instruction, or cue, to 
do so, it is seen as primarily targeting the learning function of imitation. While there is research demonstrating the effectiveness of DTT in addressing the ability to imitate (Brown et al., 2009; Miller et al., 2015; Valentino et al., 2018; Young et al., 1994), there is a concern that it does not address the propensity to imitate (i.e., social motivation or social function).

Contingent imitation is a naturalistic teaching strategy that involves following the child's lead by copying the child's motor movements, actions with objects, and vocalizations. Contingent imitation is included as a component of many naturalistic developmental behavioral interventions (NDBIs), a class of interventions that are informed by behavioral and developmental sciences (Schreibman et al., 2015). Reciprocal Imitation Training is one such NDBI, developed to address the missing aspect of the social use of imitation within the traditional behavioral approach (Ingersoll \& Schreibman, 2006). Reciprocal Imitation Training targets the spontaneous use of imitation within the context of play (Ingersoll \& Gergans, 2007) and consists of contingent imitation, linguistic mapping (adult narration of the child's actions and attentional focus), and an embedded discrete trial (modeled action, prompt, and praise). The use of Reciprocal Imitation Training has resulted in gains in young children with ASD's ability to imitate after 20-30 h of treatment (Ingersoll, 2010, 2012; Ingersoll \& Lalonde, 2010; Ingersoll et al., 2007; Ingersoll \& Schriebman, 2006). Reciprocal Imitation Training has also exhibited additional, untargeted gains in the areas of expressive language, pretend play and joint attention (Ingersoll \& Schriebman, 2006; Ingersoll \& Lalonde, 2010). However, greater play skills at onset of intervention have been hypothesized to be predictive of skill acquisition during Reciprocal Imitation Training (Ingersoll, 2010), indicating that this may not be as effective of a teaching approach for individuals with greater support needs. It may be that Reciprocal Imitation Training targets the social function of imitation and is more beneficial for individuals who have mastered the ability to imitate but are struggling in their propensity to do so (Vivanti et al., 2014).

Outside of the context of NDBIs and as a stand-alone strategy, contingent imitation has been demonstrated to increase and generalize imitative behavior of children with ASD (Field et al., 2013; Hwang \& Hughes, 2000). Kaufman and colleagues (1976) demonstrated positive outcomes when contingent imitation was incorporated into a DTT framework (Kauffman et al., 1976). However, no additional research built off this combination of contingent imitation and DTT. The goal of this study was to continue with this line of inquiry to address gaps in what we know about effectively teaching imitation to young children with ASD, particularly to young learners who have exhibited challenges in acquiring this skill. A combined approach of DTT with the developmental teaching strategy of contingent imitation may assist with targeting both the learning and social functions of imitation (Uzgiris, 1981), along with the ability and the propensity to imitate (Vivanti et al., 2014).

The purpose of this study was to assess whether imitation could be successfully taught to young children with ASD exhibiting challenges with imitation using DTT paired with contingent imitation. The research questions were: 
1. Is there a functional relationship between implementation of DTT plus contingent imitation and increases in imitation of actions with objects for children with ASD?

2. What are interventionists' perceptions of the acceptability and feasibility of DTT plus contingent imitation as an intervention package?

\section{Method}

\section{Participants}

Participants were recruited from an inclusive university-based preschool program. The first author informed teachers about the study, who then sent information home to caregivers of children for whom the teachers believed the intervention may be beneficial due to ongoing challenges with imitation. Participants were considered eligible for the study if a) they had a diagnosis or educational classification of ASD; b) their preschool teachers reported that they were not currently consistently imitating actions with objects; c) they had been working on this skill and showed minimal progress towards acquisition of imitation; and d) the individual was between 24 and 60 months of age. These broad inclusion criteria were decided upon due to public preschool programs accepting all comers and all children with disabilities having the right to a Free and Appropriate Public Education (FAPE; Individuals with Disabilities Education Act, 2004).

Three children with a diagnosis of ASD participated in the study. The children ranged in age from 4-years to 4-years, 8-months at study onset. All participant families spoke English at home. Specific data on participant socioeconomic status were not obtained.

The Motor Imitation Scale (MIS; Stone et al., 1997) was administered to quantify pre-intervention imitation performance. The MIS is a structured imitation assessment that includes eight actions with objects and eight motor tasks, resulting in a total MIS score and subscale scores for Object Imitation and Body Imitation. Actions are modeled up to three times with the instruction, "Now you do it" or "Your turn!" Participants received a score of 0 for no imitation, 1 for partial imitation, and 2 for complete imitation. For the purpose of this study, Object Imitation scores were calculated into the percent of items performed correctly and are reported below. Due to COVID 19 closures, post-intervention scores for this measure were not available and therefore these data are solely being presented to further describe participants. Specific characteristics of each participant are described below.

Kaelon is a Black male. He was diagnosed with ASD at 2 years of age and was 4 years old at study onset. Kaelon's pre-intervention MIS Object Imitation score was $25 \%$. Per teacher report, Kaelon's object play primarily consisted of stereotypical behavior, such as mouthing objects and clapping items in his hands. Kaelon was nonverbal and was learning to use an iconic communication system at study onset to request preferred objects and edible items. 
Trevor is a male of mixed racial background, Black and White. He received an ASD diagnosis at age 3 and was 4-years, 6-months at study onset. Trevor's pre-intervention MIS Object Imitation score was $43.75 \%$. Per teacher report, Trevor exhibited some appropriate object play at the beginning of the study, such as looking at books and pushing keys on a toy piano. Trevor was nonverbal and was learning to use an iconic communication system to request breaks and preferred objects at study onset.

Casey is a White male. He was diagnosed with ASD at age 2 and was 4-years, 8-months at study onset. Casey's pre-intervention MIS Object Imitation score was $31.25 \%$. Casey's object play skills primarily consisted of appropriate engagement with sensory materials such as playdough and stretchy tubes, as well as stereotypical behavior, such as holding and dropping small items in front of his eyes. Casey was nonverbal and used an iconic communication system to request preferred objects and edible items.

This study was exempted by the University of Washington Institutional Review Board under research that involves no more than minimal risk to subjects. All participants' caregivers provided informed consent prior to participation.

\section{Setting and Materials}

Participants attended their early learning program five days per week and received additional intensive instruction within an ASD specific classroom three days per week in the same program. Participants were enrolled in separate classrooms. Intervention took place three days per week within participants' ASD specific classrooms in a space separated from peers. Ten objects were used during baseline and intervention and were consistent across all participants (see Table 1).

Table 1 Actions with objects targeted during imitation trials with description

\begin{tabular}{|c|c|}
\hline Action & Description \\
\hline Block in bowl & Block is placed inside bowl (does not have to be released from hand) \\
\hline Wave streamer & $\begin{array}{l}\text { Wand moves back and then forth within } 2 \mathrm{~s} \text {, moving at least } 1 \text { inch (wand may be } \\
\text { on or off the table) }\end{array}$ \\
\hline Puts cup to mouth & Cup is brought within 1 inch of mouth (can be upside down or sideways) \\
\hline Tap table with block & $\begin{array}{l}\text { Block is tapped against table at least } 1 \text { or more time(s). Nonexample: Setting block } \\
\text { down on table }\end{array}$ \\
\hline Stir spoon in bowl & $\begin{array}{l}\text { Spoon is moved up-and-down or side-to-side in bowl (spoon can be upside down; } \\
\text { movement may be subtle) }\end{array}$ \\
\hline Push car & Car is moved across surface at least $1 \mathrm{inch}$ \\
\hline Shake bell & Bell is moved side-to-side or up-and-down and results in sound \\
\hline Tap sticks & One stick in each hand, touched together and brought apart 1 or more times \\
\hline Dots on paper & Marks 1 or more dots on the paper \\
\hline Drop bean bag & Releases bean bag from above the table or floor \\
\hline
\end{tabular}




\section{Interventionist Training}

Intervention was conducted by master's students studying applied behavior analysis who were working in the participants' classrooms. Interventionists were trained to 90\% correct implementation via written training materials, observation of the first author conducting the intervention, and feedback provided from the first author during their first several sessions.

\section{Experimental Design}

A multiple probe design was conducted across participants (Horner \& Baer, 1978). Each participant completed a minimum of five baseline sessions, and implementation of intervention was initiated with the participant with the most stable baseline at that time (Kaelon). After Kaelon's data demonstrated a trend in the desired direction, intervention was implemented with the second participant (Trevor). Once Trevor's data demonstrated a trend in the desired direction, intervention was implemented with the final participant (Casey).

\section{Dependent Variable}

The dependent variable was child independent imitation of actions with objects. Trial-by-trial data were collected and summarized as percentage correct per session across baseline and intervention. Baseline sessions consisted of 10 DTT trials, one per action with object. Actions with objects were probed one time each to limit frustration for participants via repetitive probing. Intervention consisted of 30 total DTT trials per session. These trials were completed for three actions with objects (10 trials per action) as three actions were targeted at a time during intervention to reflect current practice in DTT. Trials to criterion data were calculated at the end of intervention to evaluate possible changes in rate of mastery over time.

\section{Procedure}

\section{Preference Assessment}

Prior to baseline, preference assessments were conducted to inform hypothesized reinforcers used in the study. An indirect preference assessment was completed by participants' caregivers via the Reinforcement Assessment for Individuals with Severe Disabilities (RAISD; Fisher et al., 1996). Results were used to select materials for a direct preference assessment, which was conducted by the first author. A Free Operant preference assessment (Roane et al., 1998) was conducted with Kaelon and Casey and a Single Stimulus preference assessment (Pace et al., 1985) was conducted with Trevor. 


\section{Baseline}

Baseline sessions consisted of ten object imitation probes presented by an interventionist using a DTT format. Actions were taken from the Project DATA (Schwartz et al., 2017) and Work in Progress (Leaf \& McEachin, 1999) curricula (see Table 1). Each action was presented once per baseline session in a randomized order. No feedback was provided based on correct imitation; however, positive feedback for participation was provided between trials.

\section{Intervention}

Intervention consisted of 30 DTT trials with contingent imitation. A traditional discrete trial was completed consisting of a discriminative stimulus ("Do this," "Copy me," "Do the same," or something similar, plus performance of the action), prompts and prompt fading, and error correction. This discrete trial was immediately followed by contingent imitation; after the participant imitated independently or with assistance, the interventionist performed the action again while vocally labeling the participant's imitative behavior (e.g., "You copied me," "You did the same") prior to delivery of tangible reinforcement. This additional imitative action by the interventionist was intended to provide additional instructional feedback and to mimic a reciprocal back-and-forth imitative interaction within the structured teaching context. This was similar to the way in which contingent imitation was included with DTT in Kauffman et al.,'s 1976 study. After every set of 10 DTT plus contingent imitation trials, participants were given an opportunity to engage in free play with preferred objects for two minutes. During these "breaks," interventionists were instructed to engage in contingent imitation of the participants' play. However, this was an unmeasured aspect of the intervention procedures.

Three actions with objects were targeted at a time throughout intervention and were presented in randomized order. For example, participants were taught "block in bowl," "wave streamer," and "puts cup to mouth" together. When criterion was met on any item $-80 \%$ accuracy across two consecutive intervention sessions - that item was removed from the rotation and replaced with a new action with an object. This continued until criterion was met on all 10 actions with objects or 10 weeks of intervention had occurred. Actions with objects targeted in intervention were the same as those assessed during baseline (see Table 1).

A most-to-least prompting hierarchy was used across participants. This consisted of full physical prompts (hand over hand), partial physical prompts (manual guidance at forearm), light touch/shadow prompts, and model prompting (miming the action). For Trevor and Casey, support was decreased when they performed three consecutive actions correctly at the current prompting level. Support was increased when they performed three consecutive actions incorrectly at the current prompting level. For Kaelon, support was decreased when he performed five consecutive actions correctly at the current prompting level, and support was increased when he performed two consecutive actions incorrectly. Error correction across participants included neutral verbal feedback ("Not quite," "let's try again,"), followed by 
re-presenting the action and verbal cue to imitate and providing a more intrusive prompt to ensure correct performance.

\section{Fidelity of Implementation}

The first author and trained graduate assistants scored fidelity of implementation via videotape or live observation on $56 \%$ of baseline sessions and $27 \%$ of intervention sessions. Average fidelity of implementation during baseline was $98 \%$ across participants (range: 92-100\%). Average fidelity of implementation during intervention was $94 \%$ across participants (range: $71-100 \%$ ). Lower fidelity scores resulted from errors with the prompting hierarchy.

\section{Interobserver Agreement}

Interobserver agreement (IOA) data were obtained via videotape or live observation during a minimum of $25 \%$ of sessions across phases per participant. Across all participants, IOA was collected for $27 \%$ of baseline sessions and $31 \%$ of intervention sessions. IOA was collected by the first author and trained graduate assistants. Average IOA was $97 \%$ (range: $90-100 \%$ ) across participants during baseline. Average IOA during intervention was 93\% (range: 66-100\%) across participants. Lower IOA scores during intervention resulted from disagreement on the level of prompted correct performance exhibited by the participant (e.g., whether a full or partial physical prompt had facilitated correct performance).

\section{Social Validity}

Interventionists completed five subscales from the Usage Rating Profile-Intervention Revised (URP-IR; Briesch et al., 2013) at the end of the study to assess intervention acceptability and feasibility. These five subscales were Acceptability, Understanding, Feasibility, System Climate, and System Support. The URP-IR uses a Likert scale rating system, from 1 (strongly disagree) to 6 (strongly agree). In addition to the URP-IR subscales, interventionists responded to an opened-ended questionnaire (see Appendix A).

Lead teachers from the inclusive university-based preschool program were consulted during development of the intervention to receive feedback a priori regarding acceptability. Additional autistic and autism community members were not involved in the design of the study.

\section{Data Analysis}

Visual analysis was used to analyze the trend, level and variability of the data across phases and participants. Group means for the Acceptability, Understanding, Feasibility, System Climate, and System Support subscales of the URP-IR were 
calculated (see Fig. 2). The open-ended questionnaire was reviewed to clarify participant responses to the URP-IR subscales.

\section{Results}

\section{Intervention Data}

\section{Trial-by-Trial Data}

Kaelon. During baseline, Kaelon exhibited stable rates of imitation with correct responding ranging from $0-10 \%$ of trials. Given that each of the 10 targets were probed once per baseline session, this equated to one target correct during four out of five baseline sessions. The targets Kaelon performed correctly during baseline varied and were not targets that he went on to master in intervention. These targets were "cup to mouth" (correctly imitated during $20 \%$ of baseline sessions), stir spoon in bowl (correctly performed during $20 \%$ of baseline sessions), and shake bell (correctly performed during $40 \%$ of baseline sessions).

During intervention, Kaelon did not engage in correct, independent responding until the fourth session. Kaelon then exhibited a rapid increase in correct responding with one of the three targets (block in bowl), which he mastered after seven sessions. Throughout the remainder of intervention, Kaelon exhibited variable performance across targets, mastering one additional target (wave streamer) after 18 sessions. Overall, Kaelon participated in intervention for 10 weeks and mastered two actions with objects (see Fig. 1).

Trevor. During baseline, Trevor exhibited variable rates of imitation with correct responding ranging from $10-50 \%$ of trials. This equated to one to five targets correct during each baseline session. The targets Trevor performed correctly during baseline varied from session to session. Two targets were performed correctly during $60 \%$ of baseline sessions ("push car," "dots on paper"); two targets were performed correctly during $40 \%$ of baseline sessions ("block in bowl," "tap table with block"); and three targets were performed correctly during $20 \%$ of baseline sessions ("wave streamer," "shake bell," "stir spoon in up").

After implementation of the intervention, Trevor's data show performance within baseline range but with an increasing trend. Trevor's data show drops in responding at the introduction of new targets, followed by increases in responding. Trevor mastered targets after the third, $13^{\text {th }}, 17^{\text {th }}, 18^{\text {th }}$, and $21^{\text {st }}$ sessions. Overall, Trevor participated in intervention for 10 weeks and mastered six actions with objects (see Fig. 1).

Casey. During baseline, Casey exhibited moderately stable rates of imitation with correct responding ranging from $20-40 \%$ of trials. This equated to two to four targets correct during each baseline session. There was some consistency to the targets Casey performed correctly during baseline. "Block in bowl" was performed correctly during $88.89 \%$ of baseline sessions, and "push car" was performed correctly during 


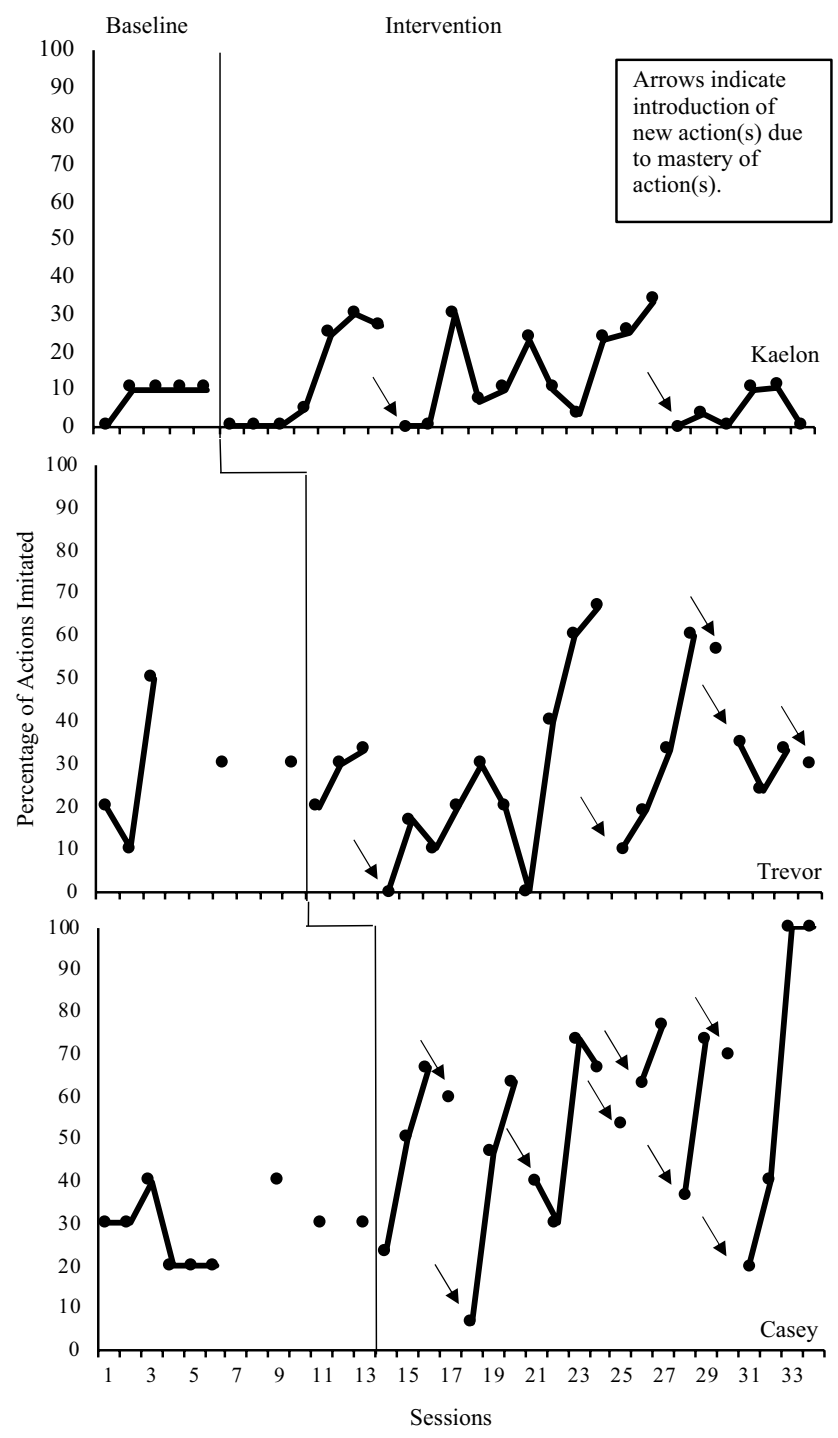

Note. Intervention data paths represent combined data for three actions targeted simultaneously. Arrows represent the introduction of a new action(s) with an object due to mastery of an action(s).

Fig. 1 Trial-by-trial data 
$77.78 \%$ of baseline sessions. The remaining targets were less consistent and were performed correctly between $0-44.44 \%$ of baseline sessions.

After implementation of the intervention, Casey's data remain consistent with baseline levels for one session, followed by an immediate increase in responding. Casey's data show drops in responding at the introduction of new targets followed by immediate increases in responding. Casey mastered targets after the third, fourth, seventh, $11^{\text {th }}, 12^{\text {th }}, 14^{\text {th }}, 16^{\text {th }}, 17^{\text {th }}$, and $21^{\text {st }}$ sessions. Overall, Casey participated in intervention for 8 weeks and mastered all 10 actions with objects (see Fig. 1).

\section{Trials to Criterion Data}

Kaelon. Kaelon's trials to criterion data show a wide range between the two mastered targets (see Table 2). The target with the lowest number of trials to mastery was inadvertently practiced within another lesson within the participant's early learning program; as such, this target's data do not reflect the true number of trials to criterion, which are unknown.

Trevor. Trevor's trials to criterion data also demonstrate a wide range across mastered targets, ranging from 30-135 trials. For the first three targets, the average trials to criterion were 88.33 . For the second three targets, the average trials to criterion were 54.33 . This represents a $38.49 \%$ reduction in trials to criterion.

Casey. Casey's trials to criterion data demonstrate a wide range across mastered targets, ranging from 30-140 trials. For the first five targets, the average trials to criterion were 68 . For the second five targets, the average trials to criterion were 41 . This represents a $39.71 \%$ reduction in trials to criterion.

Table 2 Trials to criterion data

\begin{tabular}{llll}
\hline Action with Object & Kaelon & Trevor & Casey \\
\hline 1.Block in bowl & 70 & 30 & 30 \\
2.Wave streamer & 180 & 100 & 40 \\
3.Cup to mouth & Not met & 135 & 140 \\
4.Tap table with block & Not met & 67 & 80 \\
5.Push car & Not met & 66 & 50 \\
6.Stir spoon in bowl & Not met & 30 & 30 \\
7.Shake bell & Not met & Not met & 30 \\
8.Tap sticks & Not met & Not met & 40 \\
9.Drop bean bag & Not met & Not met & 65 \\
10.Dots on paper & Not met & Not met & 40 \\
Mean Trials to Criterion & 125.00 & 71.33 & 54.50 \\
\hline
\end{tabular}

Actions with objects numbers 1-3 were introduced simultaneously to all participants. A new action with an object was introduced each time an action was mastered 


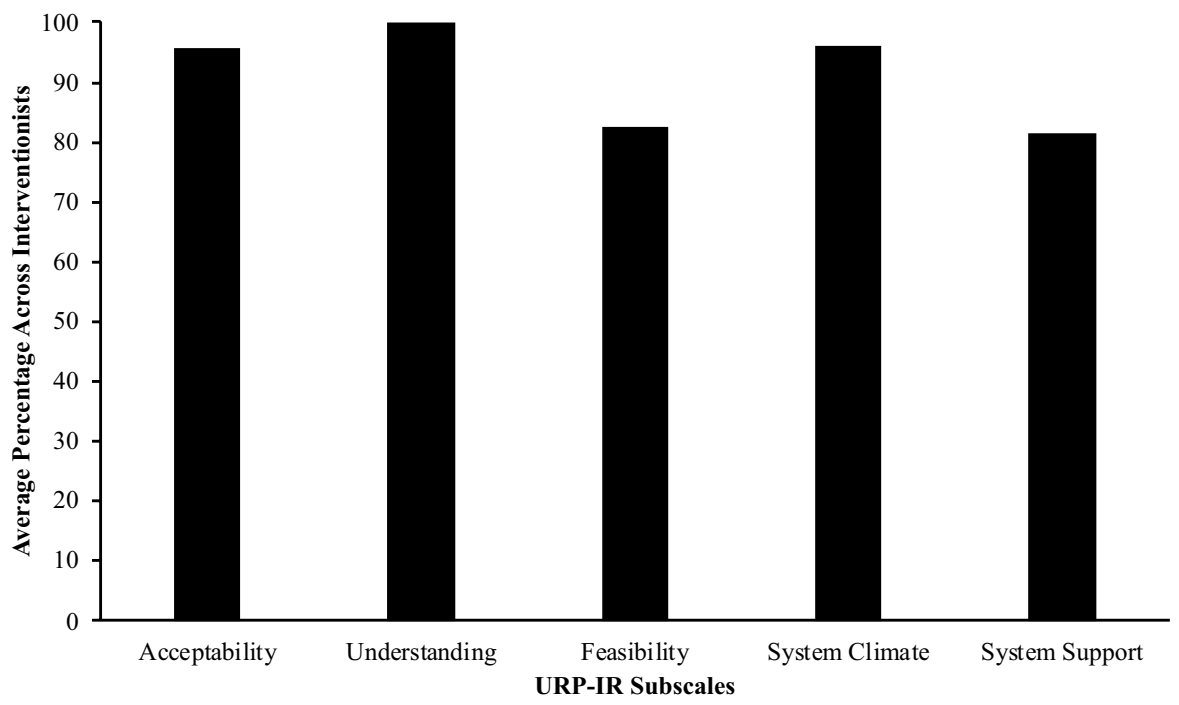

Fig. 2 URP-IR subscale scores

\section{Social Validity}

The highest URP-IR subscale rating was in Understanding (100\%), followed by System Climate (96.1\%), Acceptability (95.7\%), Feasibility (82.4\%), and System Support (81.5\%; see Fig. 2). Responses provided to the follow-up questionnaire were overwhelmingly positive. Kaelon's interventionist reported that he appeared to be more proficient in cued imitation by the end of the study. Trevor's interventionist reported that she and Trevor's preschool team observed Trevor engage in spontaneous imitation in his preschool classroom towards the end of the intervention. Casey's interventionist reported a "noticeable difference" in his joint attention during free play by the end of the study. At the same time, two of the three interventionists referenced the number of discrete trials as challenging to fit into participants' school days, and that this was the most challenging aspect of the intervention. However, one interventionist reported that the number of trials seemed important in solidifying acquisition of this skill.

Taken together, these data suggest that this intervention package was viewed in a positive manner by the early learning program interventionists.

\section{Discussion}

The purpose of this intervention was to combine DTT with contingent imitation into a user-friendly strategy for teaching imitation to young children with ASD who have struggled to acquire this skill according to their instructional team. Two participants demonstrated moderate effects with increased imitative responding while one 
participant demonstrated minimal effects with slight increases in imitative responding, indicating variable responses. One participant acquired all 10 targeted behaviors during the intervention, one acquired 6 of the 10, and one participant acquired two of the behavioral targets. Participants who entered the study with higher MIS scores and greater teacher-reported appropriate object play (Trevor and Casey) experienced greater gains. The student who acquired two actions had the lowest MIS score and was described by his teachers as lacking appropriate object play skills and primarily engaging in stereotypy (Kaelon).

Given Kaelon's low baseline level of imitating actions with objects and anecdotal limited range of object play, the ability to imitate and learning function of imitation may have been the primary areas of need for Kaelon at time of intervention. One of the challenges with implementation of intervention for Kaelon was preventing satiation on reinforcers, which likely contributed to difficulty obtaining Kaelon's full, engaged attention during intervention trials. School- and caregiver-approved edible reinforcers (graham crackers) were primarily used, alternated with access to identified preferred objects (squishy objects, light-up objects, noisemakers). However, these objects were sensitive to rapid satiation. For Kaelon, a beneficial goal may have been to expand reinforcing stimuli for use within an imitation intervention.

Trevor's variable baseline data indicate that for him the propensity to imitate and the social function of imitation may have been the primary areas of need at time of intervention. His trial-by-trial and trials to criterion data indicate an increase in his rate of responding over time, but more data are needed to determine whether this intervention would continue to improve his overall propensity to imitate. It could be that the observed increase in Trevor's responding still fell within his typical range of responding, given that he had engaged in inconsistent imitation of actions with objects during baseline.

Casey's moderate level of responding at baseline and increasing rate of mastery during intervention indicate that the propensity to imitate and social function of imitation may have been the areas of need at time of intervention. Casey's data demonstrate an increasing rate of target acquisition during intervention, indicating that he was acquiring the skills to imitate more readily and increasing the fluency with which he responded to previously untargeted stimuli. According to his interventionist, this mastery acceleration was partnered with a perceived increase in joint attention, which could highlight the importance of additional social skills when it comes to one's propensity to imitate as indicated by Vivanti et al. (2014). Similar to Trevor, while Casey's teachers had reported that he did not imitate actions with objects at study onset, his baseline data did show consistent imitative responses to specific stimuli. This further contributes to the possibility that it was the propensity, not the ability, to imitate that was challenging for Casey at study onset.

While few conclusions may be drawn regarding the efficacy of the combined intervention package based on the data, the differing outcomes across participants may underscore the importance of individualizing actions with objects targeted within cued imitation instruction. For individuals not yet engaging in appropriate object play and for whom the ability to imitate is the primary target, it could be beneficial to target actions with objects that participants have been observed to engage in spontaneously rather than arbitrary actions specified within a curriculum. 
For participants who are engaging in some appropriate object play and for whom the propensity to imitate may be the focus of intervention, this may be of lesser importance. This is consistent with prior recommendations that familiar actions are targeted in intervention prior to new actions (Ingersoll, 2008; Ledford \& Wolery, 2011).

All interventionists rated the approach favorably, and anecdotal indications of generalization and spontaneous imitation were provided by Trevor's and Casey's interventionists. Trevor's interventionist and preschool teachers reported observing Trevor engage in spontaneous imitation during classroom free play. Casey's interventionist reported an increase in joint attention. While subjective and unmeasured, this information could suggest that combining contingent imitation with DTT may support gains in untargeted skills such as joint attention. This is consistent with prior research suggesting that adult contingent imitation may increase nonverbal communication such as attention, proximity to adults, social eye gaze as well as verbal interaction (Ishizuka \& Yamamoto, 2016; Killmeyer et al., 2019).

Social validity data indicate positive responses to the intervention. Overall, interventionists reported understanding how to use the intervention, acceptability of the intervention by the early learning program, fit of intervention with other teaching methods used in their setting, and an overall improvement in participants imitative skillsets. However, social validity data also indicate that the number of targeted discrete trials was a challenge and may limit feasibility across settings.

\section{Limitations and Future Directions}

There are limitations to acknowledge with this study. Given school closures as a result of COVID-19, participants were not available for post-intervention assessment on the Unstructured Imitation Assessment (Ingersoll \& Lalonde, 2010), which measures a child's use of spontaneous imitation during play, and the Motor Imitation Scale (Stone et al., 1997), which measures a child's use of cued imitation during structured but playful interactions. As a result, the study focused on the effects of DTT paired with contingent imitation on the learning of cued imitation of actions with objects only, rather than evaluating outcomes affiliated with both cued and spontaneous imitation. COVID-19 school closures also prevented obtaining generalization and maintenance data. Given that during intervention participants did not begin to immediately imitate new actions as they were introduced, it is evident that cued imitation of actions with objects had not yet fully generalized. Future research should incorporate post-intervention assessment measures on cued and spontaneous imitation, and follow-up measures of maintenance and generalization beyond intervention sessions. Simultaneously, continuing intervention beyond the 10 -week or 10 actions with objects end point for individuals who are exhibiting positive responding may provide insight into at what point, if at all, imitative repertoires begin to generalize.

Additional individualization of imitation intervention is likely warranted to increase positive outcomes across learners and skillsets. This might include varying intervention dosage, targeting additional environments for teaching (e.g., the home), 
and individualizing the actions with objects that are targeted in intervention rather than adhering to a curriculum specifying actions to target. Further, interventionist feedback on feasibility of this study is important for future research and practice. The number of trials was described as challenging to implement given time constraints and other skills targeted within participants' early learning program. Future research should address this factor to evaluate effective instructional strategies that are adoptable and scalable. Additionally, there was no systematic home-school collaboration within this intervention. Given that imitation is important across environments, future research should include a systematic way of sharing intervention strategies with families to support generalization to the home.

Finally, there are significant limitations in the design of the study. Baseline data consisted of fewer trials per action compared with intervention. As such, it is plausible that changes in the number of actions imitated across participants could be due to repeated practice rather than the intervention itself. Future research should incorporate stronger single-case design methodology to evaluate the effects of the instructional package.

In conclusion, additional research evaluating and synthesizing effective methodologies for teaching imitation across learners with ASD with varying support needs is warranted. Although the majority of behavioral research on imitation has focused on the ability to imitate, it is clear that understanding how to promote children's propensity to imitate is also needed. To address this complicated and important skill, instructional strategies across disciplines may need to be combined to support student learning. The current study adds to the literature by presenting an intervention package to teach imitation to young learners with ASD using both behavioral (DTT) and developmental (contingent imitation) teaching strategies.

\section{Appendix A}

\section{Social Validity Questionnaire}

1. Which aspect of the intervention did you like the most? Why?

2. Which aspect of the intervention did you like the least? Why?

3. Which aspects of the intervention were the most difficult to implement? Why?

4. Which aspects of the intervention were least difficult to implement? Why?

5. Describe the outcome(s), if any, of the intervention.

6. What recommendations do you have for a teacher who is planning to teach a young learner imitation?

Funding The authors did not receive support from any organization for the submitted work.

Data Availability Research protocols available upon request from the first author. 
Code Availability Not applicable.

\section{Declarations}

Ethics Approval This study was reviewed and deemed exempt by the Institutional Review Board as the protocol was deemed to involve no more than minimal risk to participants and with the 1964 Helsinki declaration and its later amendments or comparable ethical standards.

Consent to Participate Informed consent was obtained from legal guardians.

Consent for Publication Not applicable.

Conflicts of Interest The authors have no relevant financial or non-financial interests to disclose.

\section{References}

Baer, D. M., Peterson, R. F., \& Sherman, J. A. (1967). The development of imitation by reinforcing behavioral similarity to a model. Journal of the Experimental Analysis of Behavior, 10(5), 405-416. https://doi.org/10.1901/jeab.1967.10-405

Briesch, A. M., Chafouleas, S. M., Neugebauer, S. R., \& Riley-Tillman, T. C. (2013). Usage Rating Profile--Intervention; Revised (URP-IR) APA PsycTests. https://doi.org/10.1037/t66845-000

Brown, F. J., Peace, N., \& Parsons, R. (2009). Teaching children generalized imitation skills. Journal of Intellectual Disabilities, 13(1), 9-17. https://doi.org/10.1177/1744629509103514

Field, T., Ezell, S., Nadel, J., Grace, A., Allender, S., \& Siddalingappa, V. (2013). Reciprocal imitation following adult imitation by children with autism: Reciprocal imitation. Infant and Child Development, 22(6), 642-648. https://doi.org/10.1002/icd.1812

Fisher, W. W., Piazza, C. C., Bowman, L. G., \& Amari, A. (1996). Integrating caregiver report with a systematic choice assessment. American Journal on Mental Retardation, 101, 15-25.

Horner, R. D., \& Baer, D. M. (1978). Multiple-probe technique: A variation on the multiple baseline. Journal of Applied Behavior Analysis, 11(1), 189-196. https://doi.org/10.1901/jaba.1978.11-189

Hwang, B., \& Hughes, C. (2000). Increasing early social-communicative skills of preverbal preschool children with autism through social interactive training. Journal of the Association for Persons with Severe Handicaps, 25(1), 18-28. https://doi.org/10.2511/rpsd.25.1.18

Individuals with Disabilities Education Act, 20 U.S.C. $§ 1400$ (2004).

Ingersoll, B. (2008). The social role of imitation in autism: Implications for the treatment of imitation deficits. Infants \& Young Children, 21(2), 107-119. https://doi.org/10.1097/01.IYC.0000314482. 24087.14

Ingersoll, B. (2010). Brief report: Pilot randomized controlled trial of Reciprocal Imitation Training for teaching elicited and spontaneous imitation to children with autism. Journal of Autism and Developmental Disorders, 40(9), 1154-1160. https://doi.org/10.1007/s10803-010-0966-2

Ingersoll, B. (2012). Brief report: Effect of a focused imitation intervention on social functioning in children with autism. Journal of Autism and Developmental Disorders, 42(8), 1768-1773. https://doi. org/10.1007/s 10803-011-1423-6

Ingersoll, B., \& Gergans, S. (2007). The effect of a parent-implemented imitation intervention on spontaneous imitation skills in young children with autism. Research in Developmental Disabilities, 28(2), 163-175. https://doi.org/10.1016/j.ridd.2006.02.004

Ingersoll, B., \& Lalonde, K. (2010). The impact of object and gesture imitation training on language use in children with autism spectrum disorder. Journal of Speech, Language, and Hearing Research, 53(4), 1040-1051. https://doi.org/10.1044/1092-4388(2009/09-0043

Ingersoll, B., Lewis, E., \& Kroman, E. (2007). Teaching the Imitation and Spontaneous Use of Descriptive Gestures in Young Children with Autism Using a Naturalistic Behavioral Intervention. Journal of Autism and Developmental Disorders, 37(8), 1446-1456. https://doi.org/10.1007/ s10803-006-0221-Z

Ingersoll, B., \& Schreibman, L. (2006). Teaching reciprocal imitation skills to young children with autism using a naturalistic behavioral approach: Effects on language, pretend play, and joint 
attention. Journal of Autism and Developmental Disorders, 36(4), 487-505. https://doi.org/10.1007/ s10803-006-0089-y

Ishizuka, Y., \& Yamamoto, J. (2016). Contingent imitation increases verbal interaction in children with autism spectrum disorders. Autism, 20(8), 1011-1020. https://doi.org/10.1177/1362361315622856

Kauffman, J., Snell, M., \& Hallahan, D. (1976). Imitating children during imitation training: Two experimental paradigms. Education and Training of the Mentally Retarded, 11(4), 324-332.

Killmeyer, S., Kaczmarek, L., Kostewicz, D., \& Yelich, A. (2019). Contingent imitation and young children at-risk for autism spectrum disorder. Journal of Early Intervention, 41(2), 141-158. https://doi. org/10.1177/1053815118819230

Koegel, R. L., Koegel, L. K., \& Carter, C. M. (1999). Pivotal teaching interactions for children with autism. School Psychology Review, 28(4), 576-594. https://doi.org/10.1080/02796015.1999. 12085986

Leaf, R., \& McEachin, J. (1999). A work in progress behavior management strategies and a curriculum for intensive behavioral treatment of autism. DRL Books.

Ledford, J. R., \& Wolery, M. (2011). Teaching imitation to young children with disabilities: A review of the literature. Topics in Early Childhood Special Education, 30(4), 245-255. https://doi.org/10. $1177 / 0271121410363831$

Lovaas, O. I., Freitas, L., Nelson, K., \& Whalen, C. (1967). The establishment of imitation and its use for the development of complex behavior in schizophrenic children. Behaviour Research and Therapy, 5(3), 171-181. https://doi.org/10.1016/0005-7967(67)90032-0

Metz, J. (1965). Conditioning generalized imitation in autistic children. Journal of Experimental Child Psychology, 2, 389-399. https://doi.org/10.1016/0022-0965(65)90023-8.

Miller, S. A., Rodriguez, N. M., \& Rourke, A. J. (2015). Do mirrors facilitate acquisition of motor imitation in children diagnosed with autism? Journal of Applied Behavior Analysis, 48(1), 194-198. https://doi.org/10.1002/jaba.187

Pace, G. M., Ivancic, M. T., Edwards, G. L., Iwata, B. A., \& Page, T. J. (1985). Assessment of stimulus preference and reinforcer value with profoundly retarded individuals. Journal of Applied Behavior Analysis, 18(3), 249-255. https://doi.org/10.1901/jaba.1985.18-249

Roane, H. S., Vollmer, T. R., Ringdahl, J. E., \& Marcus, B. A. (1998). Evaluation of a brief stimulus preference assessment. Journal of Applied Behavior Analysis, 31(4), 605-620. https://doi.org/10.1901/ jaba.1998.31-605

Rogers, S., Hepburn, S., Stackhouse, T., \& Wehner, E. (2003). Imitation performance in toddlers with autism and those with other developmental disorders. Journal of Child Psychology and Psychiatry, 44(5), 763-781. https://doi.org/10.1111/1469-7610.00162

Schreibman, L., Dawson, G., Stahmer, A., Landa, C., Rogers, R., McGee, S., Kasari, C., Ingersoll, B., Kaiser, A. P., Bruinsma, Y., McNerney, E., Wetherby, A., \& Halladay, A. (2015). Naturalistic developmental behavioral interventions: Empirically validated treatments for autism spectrum disorder. Journal of Autism and Developmental Disorders, 45(8), 2411-2428. https://doi.org/10.1007/ s10803-015-2407-8

Schwartz, I. S., Ashmun, J., McBride, B., Scott, C., \& Sandall, S. R. (2017). The DATA Model for teaching preschoolers with autism. Paul $\mathrm{H}$. Brookes Publishing.

Stone, W. L., Ousley, O. Y., \& Littleford, C. L. (1997). Motor imitation in young children with autism: What's the object? Journal of Abnormal Child Psychology, 25, 475-485. https://doi.org/10.1023/A: 1022685731726

The National Institute of Mental Health. (2018, March). Autism Spectrum Disorder. Retrieved February, 2019, from https://www.nimh.nih.gov/health/topics/autism-spectrum-disorders-asd/index.shtml

Toth, K., Munson, J., Meltzoff, A. N., \& Dawson, G. (2006). Early predictors of communication development in young children with autism spectrum disorder: Joint attention, imitation, and toy play. Journal of Autism and Developmental Disorders, 36(8), 993-1005. https://doi.org/10.1007/ s10803-006-0137-7.

Uzgiris, I. (1981). Two functions of imitation in infancy. International Journal of Behavioral Development, 4, 1-12. https://doi.org/10.1177/016502548100400101

Valentino, A. L., LeBlanc, L. A., \& Conde, K. A. (2018). Validation of a skills assessment to match interventions to teach motor imitation to children with autism. Learning and Motivation, 62, 67-76. https://doi.org/10.1016/j.lmot.2017.02.005

Vivanti, G. (2015). The importance of distinguishing propensity versus ability to imitate in ASD research and early detection. Journal of Autism and Developmental Disorders, 45(4), 1119-1120. https://doi. org/10.1007/s10803-014-2254-Z 
Vivanti, G., Trembath, D., \& Dissanayake, C. (2014). Mechanisms of imitation impairment in autism spectrum disorder. Journal of Abnormal Child Psychology, 42(8), 1395-1405. https://doi.org/10. 1007/s10802-014-9874-9

Young, J. M., Krantz, P. J., McClannahan, L. E., \& Poulson, C. (1994). Generalized imitation and response-class formation in children with autism. Journal of Applied Behavior Analysis, 27(4), 685697. https://doi.org/10.1901/jaba.1994.27-685

Publisher's Note Springer Nature remains neutral with regard to jurisdictional claims in published maps and institutional affiliations. 